\title{
A water retention model for compacted clays subjected to salinization and desalinization processes
}

\author{
Giulia Scelsi ${ }^{1}$, Gabriele Della Vecchia ${ }^{1, *}$, and Guido Musso $^{2}$ \\ ${ }^{1}$ Dipartimento di Ingegneria Civile e Ambientale, Politecnico di Milano, Milano, Italy \\ ${ }^{2}$ Dipartimento di Ingegneria Strutturale, Edile e Geotecnica, Politecnico di Torino, Torino, Italy
}

\begin{abstract}
Environmental actions are known to induce relevant effects on the fabric of compacted active clays, which are successfully described by adopting a double porosity framework. In particular, the role of aggregate deformation has been recognized as fundamental to interpret the water retention behavior and the transport properties. These aspects are particularly relevant in the context of clay liners, being the material cast in place in unsaturated conditions and subjected to wetting process by pore fluids characterized by a chemical composition that is different from the one of compaction. Experimental data evidence that the water retention properties of active clays evolve as a function of pore water chemistry, since for a given matric suction the mass of stored water changes with water salinity. In this paper, a double porosity water retention model is proposed, capable of reproducing the variation of matric suction with water content accounting for the salinity of pore fluid. The role of salinity changes is accounted for by a suitable evolution law for aggregate deformation, which in turn affects the inter-aggregate porosity and thus the storage properties of the material.
\end{abstract}

\section{Introduction}

Pore fluid composition strongly influences the hydromechanical behaviour of compacted active clays and its appropriate modelling is crucial for geo-environmental applications, such as waste containment barriers and cutoff walls. Compacted soils are emplaced in unsaturated conditions and the progressive saturation from the surrounding environment can take place with fluids having a significantly different chemical composition from the compaction one. The influence of the chemical composition of the pore fluid has to be considered in the design of clay barriers, since it affects the hydraulic and volumetric behaviour of the material. Experimental evidences show that the microstructure evolves differently on the basis of the wetting fluid. Compacted clays are characterized by a structure composed of aggregates, and two pore categories do exist: micropores within the clay aggregates and macropores between the aggregates. This doublestructure arrangement evolves upon chemical changes, as testified by several experimental observations [1-3]. Being the role of microfabric variations well recognized in terms of water retention behaviour [4-6], a relevant role of pore fluid composition on storage properties of clays is anticipated. Although the role of pore fluid chemistry and its link to the evolution of transport mechanisms has been modelled for saturated conditions (see, e.g. [1, 7]), it has received relatively limited attention for unsaturated conditions.
A conceptual water retention framework is here proposed to reproduce the evolution of storage properties of compacted clays. The model stems from the proposal of [6], which considers the evolution of the retention properties related to changes in intra and inter-aggregate void ratio. The original model, limited to distilled water as saturating fluid, is here enhanced introducing a suitable law capable of describing the evolution of the intra-aggregate void ratio with osmotic suction. The evolution law relies on the microstructural evidences discussed in [1].

\section{The retention model for active clays permeated with distilled water}

Water retention curves are usually expressed as a link between matric suction $s$ and a measure of the mass/volume of water in the pores. For deformable porous media, a convenient variable to express the water content is the so-called water ratio $e_{w}$, defined as the ratio between the volume of water $V_{\mathrm{w}}$ and the volume of solids $V_{s}$. Compacted clayey soils are generally modelled as double porosity media [7], the void ratio $e$ being split between an intra-aggregate void ratio $e_{\mathrm{m}}$ (which is the void ratio of the microstructure) and an inter-aggregate void ratio $e_{M}$ (i.e. the void ratio of the macrostructure). The evaluation of $e_{M}$ should consistently consider that aggregates are the deformable 'grains' of the macrostructure [8,9], however in most works $e_{M}$ is simply evaluated as the ratio between the volume of 
voids in the macrostructure and the volume of the solids. Accordingly, the water retention curve proposed in [6] is simply obtained by subdividing the water ratio into two components: the water stored in the aggregates, quantified by the intra-aggregate water ratio $e_{w m}$, and the water stored in the pores between the aggregates, quantified by the inter-aggregate water ratio $e_{W M}$ :

$e_{w}=e_{w m}+e_{w M}=e_{m}\left[\frac{1}{1+\left(\alpha_{m} s\right)^{n_{m}}}\right]^{m_{m}}+e_{M}\left[\frac{1}{1+\left(\alpha_{M} s\right)^{n_{M}}}\right]^{m_{M}}$

$e_{w m}$ is the ratio between the volume of water inside the intra-aggregate pores and the volume of solids, $e_{w M}$ is the ratio between the volume of water inside the interaggregate pores and the volume of solids and $s$ is matric suction. $\alpha_{m}, n_{m}$ and $m_{m}$ are the retention parameters of the micro-structure and $\alpha_{M}, n_{M}$ and $m_{M}$ are the retention parameters of the macro-structure. Equation (1) assumes that for each structural level a van Genuchten-type water retention relationship holds, and that equilibrium holds between the pore water potential within the micropores and within the macropores.

The air entry value, $1 / \alpha$, of the two structural levels is assume to depend on the current fabric of the soil, which in the case of active soils evolves along hydromechanical paths, as discussed in [4]. The following empirical relationships for the parameters $\alpha_{m}$ and $\alpha_{M}$ allows introducing the impact of void ratio changes on the water retention behaviour in a satisfying manner ([6]):

$$
\begin{aligned}
& \frac{1}{\alpha_{m}}=\alpha_{1}^{m} \exp \left(-\alpha_{2}^{m} e_{m}\right) \\
& \frac{1}{\alpha_{M}}=\alpha_{1}^{M} \exp \left(-\alpha_{2}^{M} \frac{e_{M}}{e}\right)
\end{aligned}
$$

where $\alpha_{1}^{m}, \alpha_{2}^{m}, \alpha_{1}^{M}$ and $\alpha_{2}^{M}$ are model parameters.

The evolution of aggregate size is thus a fundamental information required by the model, due to its impact on both $\alpha_{\mathrm{m}}$ and $\alpha_{\mathrm{M}}$. Equation (4) $([4,10,11])$ links the intraaggregate void ratio to the water ratio according to experimental evidences at the microstructural scale:

$$
e_{m}=\beta_{0} e_{w}{ }^{2}+\beta_{1} e_{w}+e_{m, 0}
$$

where $\beta_{0}$ and $\beta_{1}$ are parameters quantifying the swelling potential of the aggregates and $e_{m, 0}$ is the value of the intra-aggregate void ratio for dry conditions $\left(e_{w}=0\right)$.

The water retention model has already been validated, neglecting chemical effects, for both mediumlow activity clays (Boom Clay and Sicilian scaly clay in [6] and for active clays (FEBEX Bentonite in [12]).

\section{Modelling the influence of pore water chemical composition on water retention curve}

Active clays are also known to be very sensitive to changes in the composition and chemical concentration of the wetting fluid. Repulsion forces between clay particles in suspension are of electrochemical nature, and depend on the type of cations in solution, their concentration and on the dielectric constant of the wetting fluid. In an unsaturated air-water system, water is the wetting fluid and the dielectric constant does not change. The well-known Gouy Chapman Diffuse Double Layer (DDL) theory neglects the role of the type of cation and explains the interaction between clay particles in terms of concentration of the pore fluid. Accordingly, an increasing concentration causes a reduction of the repulsion forces, reducing the size of the micro-voids. In compacted soils, this ends up with the contraction of the aggregates and therefore with a decrease of the intraaggregate void ratio.

\subsection{Modelling the effects of pore water salinity on the intra-aggregate void ratio}

A convenient expression for the incremental volume strain of the microstructure induced by concentration changes, $\dot{\varepsilon}_{m, v o l}^{c}$ which mimics the predictions of the DDL theory in terms of incremental volume strains, has been proposed by [13].

$$
\dot{\varepsilon}_{m, v o l}^{c}=\beta_{c} \cdot \exp \left(-\alpha_{c} \cdot \pi\right) \dot{\pi}
$$

where $\alpha_{c}$ and $\beta_{c}$ are two material parameters and $\pi$ is osmotic suction, which for dilute systems can be expressed by the van't Hoff equation:

$$
\pi=i c_{S} R T
$$

being $i$ the number of constituents into which the molecule separates upon dissolution (i.e. $i=2$ for $\mathrm{NaCl}$ solutions and $i=3$ for $\mathrm{CaCl}_{2}$ solutions), $c_{\mathrm{s}}$ the molar concentration of solute particles, $R$ the universal gas constant and $T$ the absolute temperature.

Introducing the usual assumption of incompressible solid particles, the integration of equation (5) from a reference state where distilled water is the wetting fluid $(\pi=0)$ to a current state where the wetting fluid is a saline solution of osmotic suction $\pi>0$ provides the following expression for micro-structural void ratio changes due to salinization processes:

$$
e_{m}(\pi=0)-e_{m}(\pi)=(1+e) \cdot \frac{\beta_{c}}{\alpha_{c}} \cdot\left(1-\exp \left(-\alpha_{c} \cdot \pi\right)\right)
$$

Then, for a given water ratio, the corresponding intra-aggregate void ratio will decrease as long as pore fluid concentration increases. The capability of equation (7) to reproduce the influence of pore fluid chemical composition and concentration on clay microstructure was discussed in [1], which investigated the microstructure evolution of FEBEX bentonite samples subjected to salinization processes starting from the experimental data proposed in [14].

\subsection{Modelling the effects of pore water salinity on the water retention curve}

The assumption that the effects of water content and concentration on the microstructure are independent and additive leads to the incremental constitutive equation:

$$
\dot{e}_{m}(\pi, w)=\dot{e}_{m}^{c}+\dot{e}_{m}^{w}
$$


Where $\dot{e}_{m}^{c}$ and $\dot{e}_{m}^{w}$ are the increments in microscopic void ratio due to concentration and water content variations, obtained by differentiating equations (4) and (7), respectively. It is then assumed that the water retention behaviour of a clay subjected to wetting/drying processes including chemical effects can be simply modelled by starting from the retention curve to distilled water (Equation 1), but considering that aggregate size evolution could also vary with saline concentration (Equation 8). In this way, matric suction is set to depend on the chemical composition of the pore fluid via two main effects:

- Swelling induced by desalinization and shrinkage induced by salinization. When salty water is added to an unsaturated clay specimen without any volume constraint, the entity of swelling depends on the chemical concentration of the inflow fluid (see, e.g. [15]). The retention model accounts for this aspect via the dependency of $\alpha_{m}$ and $\alpha_{M}$ on void ratio evolution upon salinization/desalinization.

- Dependency of aggregate size on pore fluid chemistry. According to what discussed in Section 3.1, the higher the salt concentration of the pore fluid, the smaller the size of the aggregates. The induced fabric changes imply a different distribution of inter- and intraaggregate pores, causing a variation of the capillary storage effects. This aspect is accounted for by computing the intra-aggregate void ratio with equation (8) instead of equation (4).

Finally, in order to reproduce correctly a wetting process performed with a pore fluid that has a different saline concentration with respect to the pore fluid within the void space, a further balance equation is needed. In particular, the mass balance of the salt species is required to relate the pore fluid concentration of the wetting fluid to the molar concentration $c$ of salt in solution within the pores, which is in turn related to osmotic suction. By adding (or subtracting) a mass of solution having a fixed molar concentration $c_{s}$, the current concentration of the interstitial pore water $c$ evolves, and it is different from both its initial reference value $c_{0}$ and $c_{s}$. The mass balance of the species in solution can be written as:

$$
M_{c}=M_{c 0}+\Delta M_{c}
$$

where $M_{c}$ is the mass of salt dissolved within the sample at the current configuration, $M_{c 0}$ is the mass of salt dissolved within the sample in the initial configuration and $\Delta M_{c}$ is the mass of salt which is added/removed during the wetting/drying process.

Transport processes leading to ion migration (diffusion and advection) and changes in the cationic concentration in the pore fluid are out of the scope of this work. It is instead assumed that, at the scale of the representative volume of interest for the determination of the water retention behaviour, i.e. the laboratory specimen, equilibrium is guaranteed and the concentration is homogeneous.
Accordingly, it follows:

$$
\begin{gathered}
M_{c}=\frac{c e_{w}}{1+e} V=c e_{w} V_{s} \\
M_{c 0}=\frac{c_{0} e_{w 0}}{1+e_{0}} V_{0}=c_{0} e_{w 0} V_{s} \\
\Delta \mathrm{M}_{c}=c_{s} \Delta\left(\frac{e_{w}}{1+e} V\right)=c_{s} \Delta e_{w} V_{s}
\end{gathered}
$$

Where $e_{w 0}, e_{0}, c_{0}$ and $V_{0}$ are the initial (reference) values of water ratio, void ratio, concentration and sample volume, respectively. The current concentration $c$ can thus be expressed as a function of the current water ratio and of the concentration of the incoming fluid $c_{s}$ :

$$
c=\frac{1}{e_{w}}\left(c_{0} e_{w 0}+c_{s}\left(e_{w}-e_{w 0}\right)\right)
$$

\section{Model predictions and comparison with experimental data}

\subsection{Karnataka clay}

The predictive capabilities of the model have been firstly checked by reproducing the retention curves of a compacted clay, namely Karnakata clay, prepared at a given salt concentration and subjected to wetting with a pore fluid with a different chemical composition, as presented in [16]. Karnakata clay is an expansive clay characterized by a limit liquid $w_{L}=82 \%$, plastic limit $\mathrm{w}_{\mathrm{P}}$ $=23 \%$ and shrinkage limit $10 \%$. The clay was remoulded at a water content $\mathrm{w}=28 \%$ by adding pore fluids with different $\mathrm{NaCl}$ concentrations - 0, 0.4 M and $4 \mathrm{M}$ - and then statically compacted in $76 \mathrm{~mm}$-diameter oedometer rings at the optimum Proctor dry density $\rho_{\mathrm{d}}=$ $1.42 \mathrm{~g} / \mathrm{cm}^{3}$, corresponding to an initial void ratio $\mathrm{e}_{0}=0.91$. The specimens (remoulded with distilled water or salty solutions) were then saturated with inflow solutions at different chemical composition. Matric suction during wetting was determined with the filter paper method, while void ratio changes have been determined via the measured vertical displacement. The simulation of the whole set of retention curves reported in [16] is here shown. In particular, experimental data involve:

- Test S1: specimen remoulded with distilled water and inundated with distilled water;

- Test S2 and S3: specimens remoulded with distilled water and inundated with $\mathrm{NaCl}$ solutions ( $c_{\mathrm{s}}=0.4 \mathrm{M}$ and $c=4 \mathrm{M}$, respectively);

- Test S4: specimen remoulded with $0.4 \mathrm{M} \mathrm{NaCl}$ solution and inundated with distilled water.

Experimental data concerning the total void ratio evolution with suction were used as model input. Water retention parameters corresponding to the part of the model related just to water content variations (i.e equations $1,2,3,4$ ) have been calibrated on the S1 test. The comparison between model prediction and experimental data of the $\mathrm{S} 1$ test is shown in Figure 1 while model parameters are shown in Table 1. 
The role of pore fluid chemical composition has then been accounted for by means of Equation 8: the relevant values of $\alpha_{\mathrm{c}}=11 \mathrm{MPa}^{-1}$ and $\beta_{\mathrm{c}}=0.09 \mathrm{MPa}^{-1}$ have been determined by calibrating model predictions on the experimental data of tests $\mathrm{S} 2$ and $\mathrm{S} 3$, corresponding to specimens prepared with distilled water and then wetted with different saline solutions (Figure 1). The current molar concentration was predicted by means of equation (13), setting $c_{0}=0$. The agreement between model predictions and experimental data is remarkably good considering that the role of pore water chemistry is simply accounted for introducing just the evolution of aggregate size. The major role in the difference between simulations S1, S2 and S3 is here played by intraaggregate void ratio evolution: while the specimen saturated with distilled water (test $\mathrm{S} 1$ ) experiences an increasing aggregate size for increasing water content, S2 and S3 simulations shows a competing effect of aggregate swelling due to water intake and aggregate shrinkage due to salinization. The different evolution of aggregate size with water justifies the different retention behaviour at low suction values: being total void ratio almost constant, different aggregate size implies different inter-aggregate void ratios, whose role on water storage capacity is crucial for low matric suction.

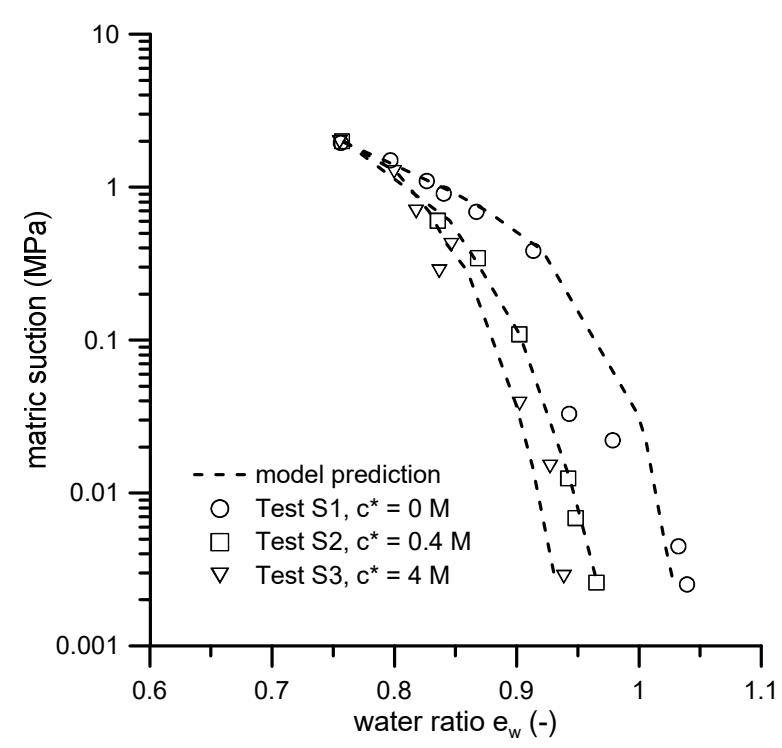

Fig. 1. Model prediction and experimental data for specimens of Karnataka clay prepared with distilled water and inundated with $\mathrm{NaCl}$ solutions at different concentration $\mathrm{c}_{\mathrm{s}}$. Experimental data from [16].

Table 1. Retention parameters for Karnataka clay.

\begin{tabular}{|c|c|c|}
\hline $\boldsymbol{\beta}_{\mathbf{0}}$ & $\boldsymbol{\beta}_{\mathbf{1}}$ & $\mathbf{e}_{\mathbf{m} \mathbf{0}}$ \\
\hline 0.25 & 0.15 & 0.35 \\
\hline
\end{tabular}

\begin{tabular}{|c|c|c|c|}
\hline $\boldsymbol{\alpha}_{\mathbf{1}}^{\mathbf{m}}$ & $\boldsymbol{\alpha}_{\mathbf{2}}^{\mathbf{m}}$ & $\mathbf{n}_{\mathbf{m}}$ & $\mathbf{m}_{\mathbf{m}}$ \\
\hline $120 \mathrm{MPa}$ & 8.9 & 3.9 & 0.03 \\
\hline
\end{tabular}

\begin{tabular}{|c|c|c|c|}
\hline $\boldsymbol{\alpha}_{\mathbf{1}}^{\boldsymbol{M}}$ & $\boldsymbol{\alpha}_{\mathbf{2}}^{\boldsymbol{M}}$ & $\mathbf{n}_{\mathbf{M}}$ & $\mathbf{m}_{\mathbf{M}}$ \\
\hline $0.09 \mathrm{MPa}$ & 11 & 0.7 & 0.055 \\
\hline
\end{tabular}

The same set of parameters identified for tests S1, S2 and S3 was then used to simulate a free swelling test performed on a salt-amended specimen remoulded with $0.4 \mathrm{M} \mathrm{NaCl}$ solution and then inundated with distilled water (test S4 in [16]). The salt mass balance equation to estimate the current chemical concentration of the pore fluid is again eq. 13 , setting $c_{s}=0$. Figure 2 shows the comparison between the predicted retention curve and the experimental data, resulting also in this case in a very satisfactory agreement.

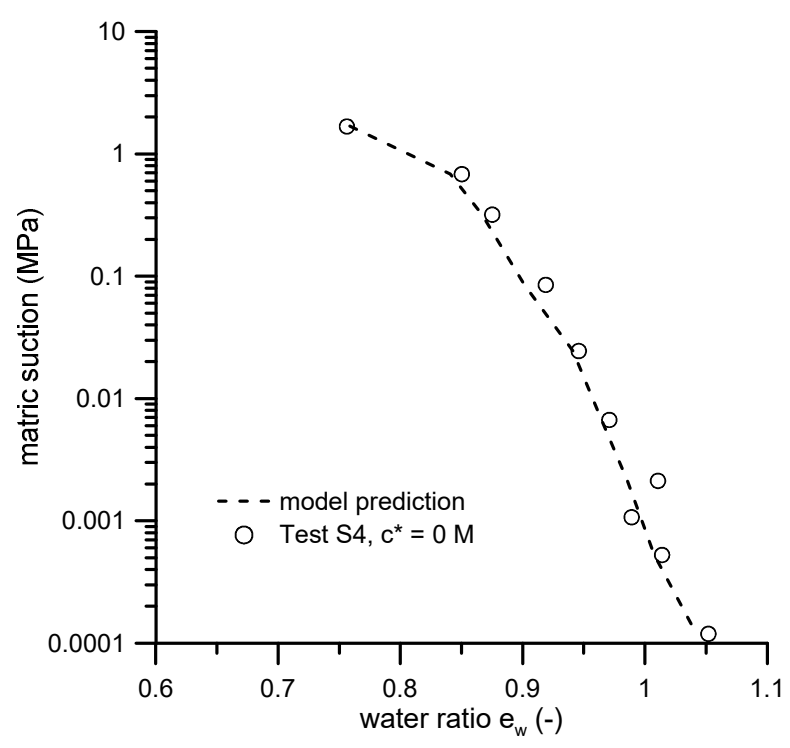

Fig. 2. Model prediction and experimental data for specimens of Karnataka clay prepared with a $c_{0}=0.4 \mathrm{M} \mathrm{NaCl}$ solution and then wetted with distilled water. Experimental data from [16].

\subsection{MX-80 bentonite}

Other experimental evidences proving the impact of the chemical composition of the pore fluid on the water retention properties have been provided in [14] for MX80 (Wyoming) bentonite. MX-80 is an American active clay $\left(\mathrm{w}_{\mathrm{L}}=350-570 \%, \mathrm{w}_{\mathrm{P}}=70 \%\right)$ mainly composed of montmorillonite (65-82\%). Compacted specimens of MX-80 bentonite have been prepared at compaction water content $w=11 \%$ and dry density $\rho_{d}=1.6 \mathrm{~g} / \mathrm{cm}^{3}$. Two specimens were then saturated in isochoric conditions with distilled water and a saline solution (osmotic suction $40 \mathrm{MPa}$ ), respectively. As expected, the larger the salt concentration of the wetting fluid, the lower the stored water content at the same matric suction. An attempt to reproduce the experimental data with the water retention model discussed above is here presented. Being the experimental results in [14] presented in terms of total suction $\psi$, experimental matric suction $s$ has been estimated as $s=\psi-\pi$, where $\pi$ is the osmotic suction corresponding to the saline solution used. Model parameters related to distilled water as pore fluid have been calibrated on the basis of the experimental data belonging to the specimen saturated with distilled water. The parameters related to aggregate swelling due to water content increase have been set equal to $e_{m 0}=0.31, \beta_{0}=0.48$ and $\beta_{1}=0.1$, 
according to [11]; the other material parameters are listed in Table 2.

Parameters $\alpha_{c}$ and $\beta_{c}$ related to aggregate size evolution with the chemical composition were obtained exploiting experimental data related to specimen saturated with brine solution. Model predictions and numerical simulations are shown in Figure 3 in terms of water ratio and matric suction. Also in this case the model seems to adequately take into account the relevant changes in clay fabric induced by the variations of the chemical concentration of the pore fluid, which end up affecting the storage properties of the material.

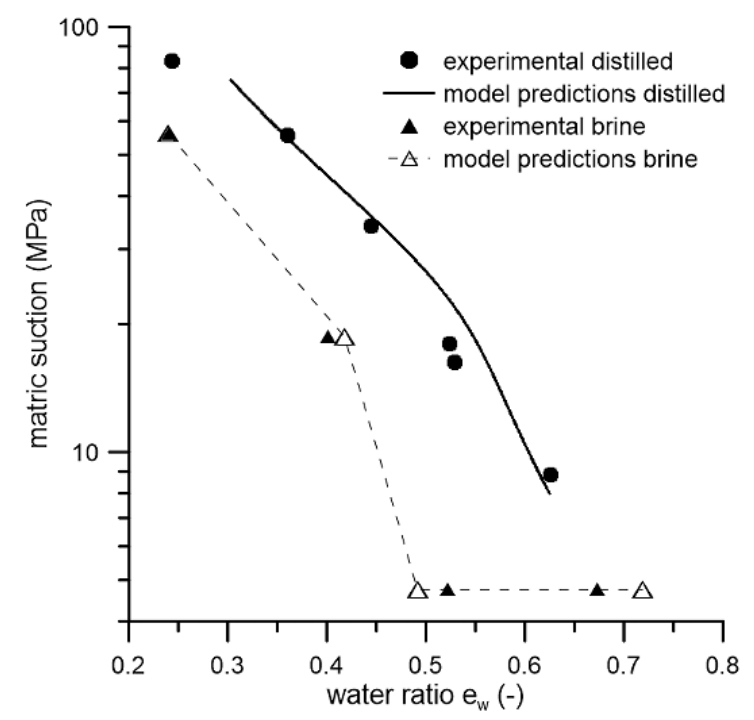

Fig. 3 - Model prediction and experimental data for specimens of MX-80 bentonite prepared with distilled water and inundated with distilled water and brine solution with osmotic suction $\pi$. Experimental data from [17].

Table 2. Retention parameters for MX-80 bentonite.

\begin{tabular}{|c|c|c|c|}
\hline $\boldsymbol{\alpha}_{\mathbf{1} \boldsymbol{m}}$ & $\boldsymbol{\alpha}_{\mathbf{2 m}}$ & $\mathbf{n}_{\mathbf{m}}$ & $\mathbf{m}_{\mathbf{m}}$ \\
\hline $200 \mathrm{MPa}$ & 4 & 7 & 0.075 \\
\hline
\end{tabular}

\begin{tabular}{|c|c|c|c|}
\hline $\boldsymbol{\alpha}_{\mathbf{M}}$ & $\boldsymbol{\alpha}_{\mathbf{2 M}}$ & $\mathbf{n}_{\mathbf{M}}$ & $\mathbf{m}_{\mathbf{M}}$ \\
\hline $10 \mathrm{MPa}$ & 10 & 9 & 0.05 \\
\hline
\end{tabular}

\section{Conclusions and discussion}

The water retention behaviour of a clay is a function, among other variables, of its microstructure. The interaction between particles of active clays is substantially ruled by environmental variables such as the water content and the chemical composition of the pore water. Necessarily, the fabric of these soils is by no means a fixed soil property, as testified by the experimental evidences about its evolution not only with water content, but also with the salinity and the dielectric constant of pore fluid. Modeling the water retention of compacted active clays then requires tracking the effects of environmental actions on clay microstructure.

A recognized way to account for fabric changes is to adopt a double porosity framework, which identifies a microscopic domain related to the clay aggregates and to the small pores within them, labeled with an intraaggregate void ratio, and a macroscopic domain, labeled with an inter-aggregate void ratio. Different water retention curves can be defined for each one of the two domains, and the overall water retention curve of the material, expressed in terms of water ratio, is given by the sum of the ones of the two domains. Environmental forces exert measurable effects mainly on the microscopic domain: aggregates swell (i.e. the intraaggregate void ratio increases) for increasing water content and decreasing concentration of dissolved species, while they shrink for opposite processes. On the other hand, the evolution of the inter-aggregate void ratio is very much dependent on the kinematic constraints imposed to the porous medium upon wetting or drying: under constant volume conditions, the macroporosity change is equal and opposite to the microporosity one, while unconstrained conditions imply different evolution patterns. Anyway, for a given water content and dry density, a higher water salinity - or osmotic suction - corresponds to a higher interaggregate void ratio. As a consequence of the evidence that the air entry value of a porous medium decreases with the amount and the size of larger pores, it follows that the matric suction associated to a given water content decreases whenever the salinity increases. Therefore, at a given density, the capacity to retain water of active clays is generally smaller when they are exposed to salty water with respect to when they are exposed to distilled water.

For modelling purposes, it was here assumed that the effects of water content and water salinity on the intraaggregate void ratio can be superimposed and calibrated separately. Constitutive laws describing the evolution of the aggregate size have been introduced reinterpreting literature data that reported experimental evidence at the microstructural level. Water retention tests were modelled taking into account the type of water used during the tests, as well as the evolution of the average salt concentration within the pore water. For wetting processes, this might induce non-monotonous histories of the intra-aggregate void ratio evolution, e.g. dominated first by the retraction due to the penetration of a high salinity water and then by the expansion due to the increase of water content. Altogether, the proposed model was found to reproduce very well water retention tests on compacted active clays as available in the literature. As for engineering aspects, when an accurate prediction of the liner behaviour is of concern (as e.g. in the case of the disposal of nuclear waste or other hazardous material of high toxicity), the present model might be coupled with a transport model predicting the changes in pore fluid composition to provide reliable simulations of pollutant migration and of liner performance. 


\section{References}

1. G. Musso, E. Romero, G. Della Vecchia, Géotechnique 63, 3 (2013)

2. T. Thyagaraj, U. Salini, Géotechnique 65, 11 (2015)

3. N. Mokni, E. Romero, S. Olivella, Géotechnique 64, 9 (2014)

4. E. Romero, G. Della Vecchia, C. Jommi, Géotechnique 61, 4 (2011)

5. F. Casini, J. Vaunat, E. Romero, A. Desideri, Acta Geotechnica 7, 2 (2012)

6. G. Della Vecchia, A.C. Dieudonné, C. Jommi, R. Charlier, Int. J. Num. An. Met. Geomec. 39, 7 (2015)

7. A. Gens, E.E. Alonso, Can. Geotech. J. 29, 10131032 (1992)

8. D. Mašín, Eng. Geol. 165, 73-88 (2013)

9. G. Musso, A. Azizi, C. Jommi, Can. Geotech. J., https://doi.org/10.1139/cgj-2019-0176

10. G. Della Vecchia, G. Musso, Soils Found. 56, 5 (2016)

11. A.C. Dieudonné, G. Della Vecchia, R. Charlier, Can. Geot. J. 54, 7 (2017)

12. G. Della Vecchia, G. Scelsi, G. Musso, It. Geotech. J. 3, 16-29 (2019) https://dx.doi.org/10.19199/2019.3.0557-1405.016

13. E. Alonso, A. Gens, W.Y. Gehling, in Proc. 3rd Eu. Conf. Num. Meth. in Geot. Eng., 11-18 (1994)

14. E. Castellanos, M.V. Villar, E. Romero, A. Lloret, Phys. Chem. Earth, Parts A/B/C 33, 516-526.

15. S.M. Rao, P. Shivananda, Can. Geotech. J. 42, 1 (2005)

16. T. Thyagaraj, S.M. Rao, J. Geotech. Geoenv. Eng. 136, 12 (2010)

17. Z. Li, G. Su, Q. Zheng, T.S. Nguyen, Acta Geotechnica, 1-19 https://doi.org/10.1007/s11440-019-00762-5 